\title{
Reconciling dark matter, solar and atmospheric neutrinos
}

\author{
J.T. Peltoniemi ${ }^{1}$ and J.W.F. Valle ${ }^{2}$ \\ Instituto de Física Corpuscular - C.S.I.C., Departament de Física Teòrica, Universitat de València, \\ 46100 Burjassot, València, Spain
}

Received 4 March 1993

(Revised 18 May 1993)

Accepted for publication 26 May 1993

\begin{abstract}
We present models that can reconcile the solar and atmospheric neutrino data with the existence of a hot dark matter component in the universe. This dark matter is a quasi-Dirac neutrino whose mass $m_{\mathrm{DM}}$ arises at the one-loop level. The solar neutrino deficit is explained via nonadiabatic conversions of $\nu_{\mathrm{e}}$ to a sterile neutrino $\nu_{\mathrm{s}}$ and the atmospheric neutrino data via maximal $\nu_{\mu}$ to $\nu_{\tau}$ oscillations generated by higher order loop diagrams. For $m_{\mathrm{DM}} \sim 30 \mathrm{eV}$ the radiative neutrino decay can lead to photons that can ionize interstellar hydrogen. In one of the models one can have observable $\nu_{\mathrm{e}}$ to $\nu_{\tau}$ oscillation rates, with no appreciable $\nu_{\mu}$ oscillations at accelerator experiments. In addition, there can be observable rates for tau number violating processes such as $\tau \rightarrow 3 \mathrm{e}$ and $\tau \rightarrow \mathrm{e}+\gamma$. In the other model one can have sizeable $\nu_{\mathrm{e}}$ to $\nu_{\mu}$ oscillation rates, as well as sizeable rates for muon number violating processes such as $\mu \rightarrow \mathrm{e}+\gamma$, $\mu \rightarrow \mathrm{e}+$ majoron and $\mu \rightarrow 3 \mathrm{e}$.
\end{abstract}

\section{Introduction}

The existing hints in favour of nonzero neutrino masses include the existence of a hot dark matter component as recently suggested by COBE data and the deficits observed in the solar $\nu_{\mathrm{e}}$ and atmospheric $\nu_{\mu}$ fluxes [1-6]. Neutrino oscillations would provide the most attractive explanation of these fluxes, which seem to be in conflict with standard theoretical expectations $[7,8]$. A common understanding of these data seems problematic, even at the level of simultaneously fitting the three phenomena. For example, one could have $\nu_{\mathrm{e}} \rightarrow \nu_{\mu}$ oscillations in the sun with $\delta m^{2} \sim 10^{-5} \mathrm{eV}^{2}$, with the $\nu_{\tau}$ as the hot dark matter (HDM) component $\left(m_{\nu_{\tau}} \sim\right.$ few $\mathrm{eV})$. However, in this scenario there is no room for oscillations to account for the

${ }^{1}$ E-mail 16444 ::PELTONIE or PELTONIE@EVALVX. Present address: SISSA, 34100 Trieste, Italy.

2 E-mail $16444::$ Valle or VALLE@EVALUN11 
atmospheric neutrino anomaly. Another possibility is again to have $\nu_{\mathrm{e}} \rightarrow \nu_{\mu}$ oscillations in the sun, while $\nu_{\mu} \rightarrow \nu_{\tau}$ oscillations are responsible for the understanding of the atmospheric neutrino data. In this case there is no room for hot dark matter.

This suggests the need for the existence of an additional light neutrino state, which must be sterile in order not to affect the invisible $\mathrm{Z}$ width, successfully predicted in the standard model.

In this paper we propose an alternative scenario that includes such a sterile neutrino $\nu_{\mathrm{s}}$. In this model the neutrino mass scales required for the joint explanation of the HDM, the solar and the atmospheric neutrino data arise from radiative corrections associated to new Higgs bosons at the electroweak scale. More than just a fit, our model provides an underlying theory that successfully explains the origin of these scales. Unlike the case of seesaw models [9], we relate the small $m_{\odot} / m_{\mathrm{D} M}$ ratio to a quantum mechanical loop suppression factor. The dark matter mass parameter $m_{\mathrm{DM}}$ arises at the one-loop level, while the oscillations responsible for the explanation of the solar and atmospheric neutrino deficits arise only at two and three loops.

In our model the HDM consists of a quasi-Dirac neutrino made mainly of $\nu_{\tau}$ and $\tau_{\mu}$. One would get the best fit to the observations by choosing its mass to be about $3 \mathrm{eV}$ leading to $\Omega_{v} \sim 0.3$ [10]. In fact, a $3 \mathrm{eV}$ Dirac-neutrino might fit the observations even better than the usually considered $7 \mathrm{eV}$ Majorana-neutrino, as it would give more power on the largest scales. On the other hand, if the HDM mass is chosen as $m_{\mathrm{DM}} \sim 30 \mathrm{eV}$ the photons produced in the radiative decays, $\nu_{\mathrm{DM}} \rightarrow \nu$ $+\gamma$ have just the right properties requires in order to ionize interstellar hydrogen, as suggested by observation [11]. The solar neutrino data are explained via the MSW effect involving nonadiabatic $\nu_{\mathrm{e}} \rightarrow \nu_{\mathrm{S}}$ transitions, while the atmospheric neutrino data can be explained via $\nu_{\mu} \rightarrow \nu_{\tau}$ oscillations with maximal mixing and $\delta M^{2} \sim 10^{-2}-10^{-3} \mathrm{eV}^{2}$. These values are in agreement with the data of the Kamiokande group and with some of the IMB studies, although not those involving stopped muons [6].

There can also be observable $\nu_{\mathrm{e}} \rightarrow \nu_{\tau}$ oscillation rates, that could be probed as a byproduct of $\nu_{\mu}$ to $\nu_{\tau}$ searches at the new generation of neutrino oscillation experiments [12]. There are, however, no appreciable $\nu_{\mu}$ to $\nu_{\mathrm{e}}$ or $\nu_{\mu}$ to $\nu_{\tau}$ oscillations on scales that can be presently probed by accelerator experiments. The model also leads to tau number violating processes such as $\tau \rightarrow 3 \mathrm{e}$ and $\tau \rightarrow \mathrm{e}+\gamma$ with rates that can lie within the experimental sensitivities of the next generation of experiments.

In an alternative model based on the $\mathrm{e}-\tau+\mu$ symmetry the experimentally observable oscillations rates are for $\nu_{\mathrm{e}} \rightarrow \nu_{\mu}$, and similarly lepton flavour violating processes now are $\mu \rightarrow \mathrm{e}+\gamma, \mu \rightarrow 3 \mathrm{e}$ and $\mu \rightarrow \mathrm{e}+\mathrm{J}$ (J denotes the majoron), with rates that can lie within the present experimental sensitivities.

Both models are compatible with laboratory, astrophysical and cosmological observations, including primordial nucleosynthesis limits. 


\section{Neutrino masses and mixing}

The existing hints from HDM, solar and atmospheric neutrino observations restrict the form of the neutrino mass and mixing. As discussed above, in order to provide a common interpretation of these data one requires the existence of a sterile neutrino. If we restrict ourselves to matrices that have a simple underlying symmetry, we have two interesting possibilities to consider: either we choose the sterile neutrino to be at the HDM scale or at the MSW scale. The first possibility was considered in ref. [13]. Here we focus on the alternative logical possibility that the sterile neutrino is at the solar neutrino mass scale. We also require the existence of a symmetry defined so that the HDM scale is invariant, while the solar and atmospheric neutrino oscillations arise as breaking effects. Our strategy follows closely the lines of refs. [14,15].

We may write the neutrino mass matrix in the following form

$$
M_{\nu}=\left(\begin{array}{cccc}
\mu & a_{\mathrm{e}} & a_{\mu} & a_{\tau} \\
a_{\mathrm{e}} & \epsilon_{\mathrm{ee}} & m & \epsilon_{\mathrm{e} \tau} \\
a_{\mu} & m & \epsilon_{\mu \mu} & M \\
a_{\tau} & \epsilon_{\mathrm{e} \tau} & M & \epsilon_{\tau \tau}
\end{array}\right),
$$

where the basis is $\left(\nu_{\mathrm{s}}, \nu_{\mathrm{e}}, \nu_{\mu}, \nu_{\tau}\right){ }^{*}$. For values of the entries $m, M \gg \mu, a_{i}, \epsilon_{i j}$ this matrix has an approximate $\mathrm{e}-\mu+\tau$ symmetry.

The neutrino mass eigenstates are given in terms of the weak eigenstates as

$$
\begin{aligned}
& \nu_{1} \approx \cos \theta_{\mathrm{m}} \cos \theta \nu_{\mathrm{e}}-\cos \theta_{\mathrm{m}} \sin \theta \nu_{\tau}+\sin \theta_{\mathrm{m}} \nu_{\mathrm{s}}, \\
& \nu_{2} \approx-\sin \theta_{\mathrm{m}} \cos \theta \nu_{\mathrm{e}}+\sin \theta \sin \theta \nu_{\tau}+\cos \theta \nu_{\mathrm{s}}, \\
& \nu_{3} \approx \frac{1}{2} \sqrt{2} \cos \theta \nu_{\tau}+\frac{1}{2} \sqrt{2} \sin \theta \nu_{\mathrm{e}}+\frac{1}{2} \sqrt{2} \nu_{\mu}, \\
& \nu_{4} \approx \frac{1}{2} \sqrt{2} \cos \theta \nu_{\tau}+\frac{1}{2} \sqrt{2} \sin \theta \nu_{\mathrm{e}}-\frac{1}{2} \sqrt{2} \nu_{\mu},
\end{aligned}
$$

where now $\theta$ denotes the mixing between $\nu_{\mathrm{e}}$ and $\nu_{\tau}$, and $\theta_{\mathrm{m}}$ is the mixing of the lightest states relevant for the solar neutrino deficit.

For suitable choices of parameters obeying this hierarchy the heaviest neutrino is quasi-Dirac, formed by $\nu_{\mu}$ and $\nu_{\tau}$ and its mass $m_{\mathrm{DM}}$ can be chosen to be at the HDM scale. The remaining neutrinos have much smaller masses at the MSW scale, and their mixing can explain the deficit of solar $\nu_{\mathrm{e}}$ neutrinos. Finally, the splitting between $\nu_{\mu}$ and $\nu_{\tau}$ generates oscillations that can explain the observed $\nu_{\mu}$ deficit in the atmospheric neutrino flux.

\footnotetext{
* Another phenomenologically viable alternative, considered in sect. 6 , corresponds to having the same matrix but in the reversed basis $\left(\nu_{\mathrm{s}}, \nu_{\mathrm{e}}, \nu_{\tau}, \nu_{\mu}\right)$.
} 
Neutrino oscillations are characterized by three different oscillation lengths. The shortest one is due to the mass squared difference between the heavier and lighter states, $m_{\mathrm{DM}}^{2}$, while the two long-scale oscillations are due to the squared mass difference of the lightest states $\delta m^{2}$, responsible for the explanation of the solar neutrino data, and that of the heaviest states, denoted by $\delta M^{2}$, chosen to fit the atmospheric neutrino data.

For typical energies, the oscillations at scale $m_{\mathrm{DM}}^{2}$ have oscillation lengths that can lie in the region of sensitivity of accelerator experiments and their rates could be observable. The only significant oscillation at this level occurs between electron and tau neutrinos, and is characterized by a mixing angle $\theta$. The oscillations of muon neutrinos remain experimentally unobservable in accelerator based experiments.

The oscillations to the sterile neutrino $\nu_{\mathrm{s}}$ are too small to be observed in laboratory, but they could lead to important effects in astrophysics and cosmology. Indeed, $\nu_{\mathrm{e}}, \nu_{\mu}$ or $\nu_{\tau}$ conversions could populate the sterile states $\nu_{\mathrm{s}}$ in the early universe thus increasing the effective light neutrino number and consequently the primordial light element abundances. The most dangerous transitions are those from $\nu_{\mu}$ or $\nu_{\tau}$ to the sterile neutrino, characterized by a squares mass difference $m_{\mathrm{DM}}^{2}$. In order to prevent the overpopulation of the sterile neutrinos the mixing squared between the heavy neutrinos and the sterile one should be less than about $3 \times 10^{-6}$, for $m_{\mathrm{DM}} \sim 3 \mathrm{eV}$ [16], where we have adopted the nucleosynthesis limit $\Delta N_{\nu}<0.4$. This would transform into a limit for the mass entries,

$$
a_{\mu}, a_{\tau} \cos \theta+a_{\mathrm{e}} \sin \theta \leq 0.002 \mathrm{eV} .
$$

Of all the oscillations $\nu_{i} \rightarrow \nu_{\mathrm{s}}$ with solar neutrino scale $\delta \mathrm{m}^{2}$, the only one that could be important once the above limits are fulfilled is from $\nu_{\mathrm{e}}$ into $\nu_{\mathrm{s}}$. This is precisely the channel that is responsible for the explanation of the solar neutrino data by the MSW effect and is characterized by the mixing angle $\theta_{\mathrm{m}}$. The nucleosynthesis bound implies that these solar neutrino conversions must take place in the nonadiabatic regime, excluding the large mixing solution.

The oscillation from $\nu_{\mu}$ to $\nu_{\tau}$ is important for the explanation of atmospheric neutrinos. Due to the assumed symmetry structure, this oscillation is characterized by almost maximal mixing. In order to account for the deficit of muon neutrinos observed in contained events at underground detectors (IMB, Kamiokande and Soudan2) one should have $\delta M^{2} \sim 10^{-2}-10^{-3} \mathrm{eV}^{2}$. From the point of view of our models, it is hard to account for a smaller value of the mixing would be indicated by the most recent and controversial data on up-going muons from IMB and Baksan.

\section{A model}

We now briefly describe a model that naturally embodies the structure described above, allowing a simple explanation of the HDM, solar and atmospheric 
TABLE 1

$\mathrm{SU}(2) \otimes U(1)_{\mathrm{Y}} \otimes \mathrm{U}(1)_{\mathrm{G}}$ assignments of the leptons and Higgs scalars in the model of sect. 3.

\begin{tabular}{cccc}
\hline & $\underline{T}_{3}$ & $Y$ & $G$ \\
\hline$\Psi_{\mathrm{L} \ell}$ & $\frac{1}{2}$ & -1 & 1 \\
$\Psi_{\mathrm{L} \mu}$ & $\frac{1}{2}$ & -1 & -1 \\
$\ell_{\mathrm{R}}$ & 0 & -2 & 1 \\
$\mu_{\mathrm{R}}$ & 0 & -2 & -1 \\
$\nu_{\mathrm{s}}$ & 0 & 0 & $-\frac{1}{2}$ \\
$\phi$ & $\frac{1}{2}$ & 1 & 0 \\
$\bar{\phi}$ & $\frac{1}{2}$ & 1 & 0 \\
$\mathrm{~s}^{-}$ & 0 & -2 & 0 \\
$\eta^{-}$ & 0 & -2 & $\frac{3}{2}$ \\
$\kappa^{--}$ & 0 & -4 & 2 \\
$\Xi^{--}$ & 0 & -4 & $\frac{5}{2}$ \\
$\sigma$ & 0 & 0 & $\frac{1}{2}$ \\
\hline
\end{tabular}

Another model was discussed in sect. 6. In addition, for each case one can obtain variant models by exchanging the signs of the $\mathrm{U}(1)_{\mathrm{G}}$ charges of $\nu_{\mathrm{s}}, \eta, \kappa, \Xi$ and $\sigma$. Both models can be formulated with explicit or spontaneous breaking of the $\mathrm{U}(1)_{\mathrm{G}}$ symmetry. Only in the latter case is the scalar $\sigma$ present.

neutrino puzzles. The lagrangian is given by

$$
\begin{aligned}
& \sum_{i, j} h_{i j} \bar{\Psi}_{\mathrm{L} i} \phi l_{\mathrm{R} i}+f_{\mathrm{e} \mu} \Psi_{\mathrm{e}}^{\mathrm{T}} C i \tau_{2} \Psi_{\mu} s^{+}+f_{\mu \tau} \Psi_{\mu}^{\mathrm{T}} \mathrm{Ci} \tau_{2} \Psi_{\tau} s^{+} \\
& +\xi_{\mathrm{e}} \bar{e}_{\mathrm{R}} \nu_{\mathrm{s}} \eta^{-}+\xi_{\tau} \bar{\tau}_{\mathrm{R}} \nu_{s} \eta^{-}+\sum_{\ell, \ell^{\prime}=\mathrm{e}, \tau} g_{\ell \ell^{\prime}} \ell_{\mathrm{R}}^{\mathrm{T}} C l_{\mathrm{R}}^{\prime} \kappa^{++} \\
& +M_{\mathrm{s} \phi \bar{\phi}} \phi^{\mathrm{T}} \tau_{2} \tilde{\phi} s^{+}+M_{\mathrm{s} \eta \kappa^{-}} s^{+} \eta^{+} \kappa^{--} \\
& \quad+M_{\eta \eta \Xi} \eta^{+} \eta^{+} \Xi^{--}+M_{\kappa \Xi}^{2} \Xi^{--} \kappa^{++}+\text {h.c. }
\end{aligned}
$$

The quantum numbers are summarized in table 1. The terms on the first line in the above equation generate the entries $m$ and $M$ in eq. (1) at the one-loop level, as a result of electroweak breaking (see fig. 1a) and may be written as $[17,18]$

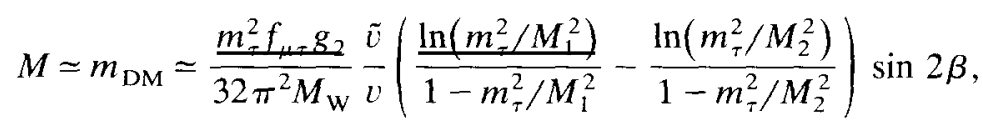

where $g_{2}$ is the SU(2) gauge coupling, $M_{1}$ and $M_{2}$ are the physical masses of the relevant single charged Higgs, their mixing angle is denoted $\beta$, and $\nu$ and $\tilde{\nu}$ denote the vacuum expectation values of the two Higgs doublets. These parameters can easily be chosen so as to provide the mass of the HDM neutrino. The mixing of this dark matter neutrino (see below) is determined from

$$
\tan \theta \simeq \frac{m}{M} \simeq \frac{m_{\mu}^{2}}{m_{\tau}^{2}} \frac{f_{\mu \mathrm{e}}}{f_{\mu \tau}} .
$$



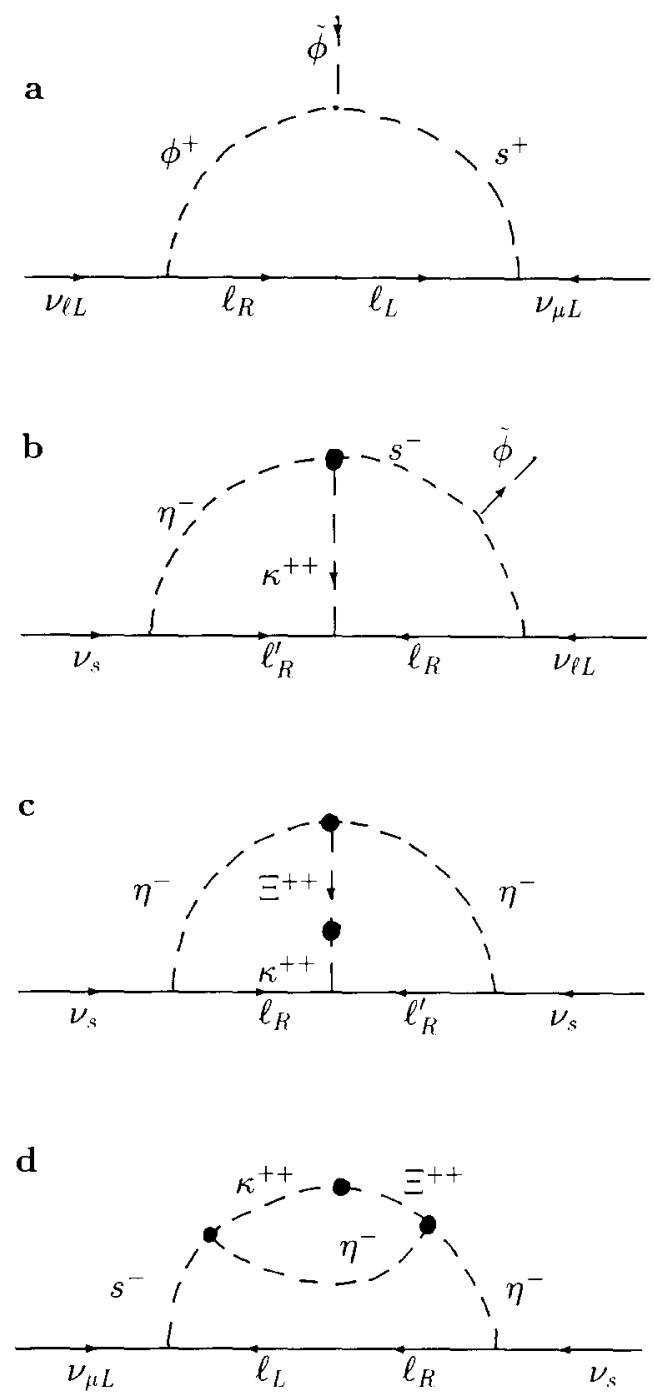

Fig. 1. Diagrams generating neutrino masses from radiative corrections (here $\ell, \ell^{\prime}=\mathrm{e}, \tau$ ). The blobs denote explicit $\mathrm{e}-\mu+\tau$ soft breaking terms.

In the absence of the last three terms of eq. (7) the model would have an exact global symmetry corresponding to $\mathrm{e}-\mu+\tau$ conservation. We can imagine that either these are small soft breaking terms (dimension less than or equal to three) or that, alternatively, a new singlet field $\sigma$ with the appropriate charge is introduced. In the latter case the symmetry would be broken spontaneously by the vacuum expectation value $\langle\sigma\rangle$ thus generating a majoron given by $J=\operatorname{Im} \sigma$. The spontaneous violation of this global symmetry at or below the electroweak scale is not in conflict with existing observations, including astrophysics and cosmology. 
For example, the coupling of the majoron to electrons does not lead to excessive stellar energy loss [19]. Moreover, the decay $\nu_{\mathrm{DM}} \rightarrow \nu_{\text {light }}+J$ can be arranged so that the resulting lifetime is longer than the age of the universe $\tau \sim 10^{17} \mathrm{sec}$. This ensures that the HDM neutrinos are still there to form the dark matter.

Whichever way this symmetry is broken, the other entries in eq. (1) will be generated by higher order, two and three loop diagrams. For definiteness we will assume in what follows that the global $G$ symmetry is broken explicitly by soft terms quadratic and cubic, as in eq. (7). As a result these entries are naturally small. In addition, they have definite charges under the $G$ symmetry given by: $\mu \rightarrow-1, a_{\mathrm{e}}, a_{\tau} \rightarrow \frac{1}{2}, a_{\mu} \rightarrow-\frac{3}{2}, \epsilon_{\mathrm{ee}}, \epsilon_{\mathrm{e} \tau}, \epsilon_{\tau \tau} \rightarrow 2$ and $\epsilon_{\mu \mu} \rightarrow-2$. In contrast, the entries $m$ and $M$ are invariant under the $U(1)_{\mathrm{G}}$ symmetry.

The values of the small mass matrix elements $a_{i}, \mu$ may be obtained from the two-loop diagram in fig. 1 , while the $\epsilon_{i j}$ arise only at the three-loop level. They may be estimated as

$$
\begin{aligned}
& \mu \sim \sum_{\alpha \beta=\mathrm{e} r} \frac{\xi_{\alpha} \xi_{\beta} g_{\alpha \beta} M_{\eta \eta \Xi} M_{\kappa \Xi}^{2}}{256 \pi^{4} M_{0}^{2}} \\
& a_{\ell} \sim \sum_{\alpha=\mathrm{e} r} \frac{m_{1} \xi_{1} M_{-} M}{256 \pi^{4} M_{0}^{2}} \frac{\tilde{v}}{v},
\end{aligned}
$$

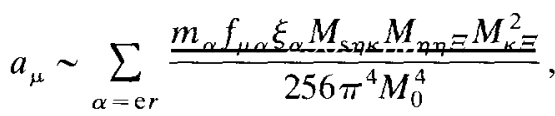

$$
\begin{aligned}
& \epsilon_{\ell \ell} \sim \frac{m_{\ell} m_{\ell}^{\prime} g_{\ell \ell} M_{\mathrm{s} \eta \kappa}^{2} M_{\eta \eta} M_{\kappa}^{2} \tilde{v}^{2} M_{\mathrm{s} \phi \phi}^{2}}{2048 \pi^{6} M_{0}^{8} v^{2}}, \\
& \epsilon_{\mu \mu} \sim \sum_{\alpha \beta} \frac{m^{2} f_{\beta \beta} g_{\alpha \beta} M^{2} M_{n} M_{*}^{2}}{2048 \pi^{6} M_{0}^{6}},
\end{aligned}
$$

where $\ell=\mathrm{e}, \tau$, and $M_{0}$ is a typical scalar mass.

It is easy to choose the parameters so that they can account for the dark matter and solar neutrino masses and mixing $\left(\delta m^{2} \sim 3 \times 10^{-6} \mathrm{eV}^{2}, \sin ^{2} 2 \theta_{\mathrm{m}} \sim 0.007\right)$, while $\delta M^{2}$ lies in the range suggested by some atmospheric neutrino observations $\left(10^{-3}-10^{-2} \mathrm{eV}^{2}\right)$. For example, one could choose $f_{\mathrm{e} \mu}=10^{-3}, f_{\mu \tau}=10^{-4}, g_{\mathrm{ee}}=$ $0.05, g_{\tau \mathrm{e}}=0.005, g_{\tau \tau}=0.001, \xi_{\mathrm{e}}=5 \times 10^{-5}, \xi_{\tau}=10^{-5}, M_{\mathrm{s} \phi \bar{\phi}}=2 \mathrm{GeV}, M_{\kappa} \equiv 20$ $\mathrm{GeV}, M_{\text {s } \eta \kappa}=M_{\eta \eta \Xi}=200 \mathrm{GeV}, M_{0}=45 \mathrm{GeV}, v=200 \mathrm{GeV}, \tilde{v}=130 \mathrm{GeV}, \sin \beta$ $=0.3$.

Note that it is also possible to obtain a variant model by exchanging the signs of the $\mathrm{U}(1)_{\mathrm{G}}$ charge assignment for $\nu_{\mathrm{s}}, \eta, \kappa$ and $\Xi$. If the scalar $\sigma$ were present, its $\mathrm{G}$ assignment would also be reversed in this new model. In what follows we will, for simplicity, not consider this variant. 


\section{Magnetic moments and radiative decay}

The transition magnetic moment matrix of neutrinos is generated by graphs similar to those that generate the mass matrix, but with a photon line inserted. The entry connecting electron and muon neutrinos is given by [18]

$$
\mu_{\nu_{\mu} \nu_{\mathrm{e}}} \simeq \frac{e m_{\mu}^{2} f_{\mu \mathrm{e}} g_{2}}{16 \pi^{2} M_{1}^{2} M_{\mathrm{W}}} \frac{\bar{v}}{v} \sin 2 \beta\left[\frac{1-r^{2}}{r^{2}}\left(1-\ln \frac{M_{1}^{2}}{m_{\mu}^{2}}\right)-\frac{1}{r^{2}} \ln r^{2}\right],
$$

where $r=M_{2} / M_{1}$.

Although the individual components of the transition magnetic moment matrix are approximately proportional to the same entries in the mass matrix, the full matrices are not. Indeed, on very general grounds one known that the mass matrix of two-component neutrinos is symmetric, while the magnetic moment matrix is antisymmetric [20]. Moreover the form factors are different and it is easy to check that, after suitable rotation in the e $\tau$ plane, there remains a nonzero transition moment between the heavy and light mass eigenstates

$$
\mu=\mu_{\mathrm{e} \mu} \cos \theta+\mu_{\mu \tau} \sin \theta
$$

In terms of the neutrino mass it can be given by

$$
\mu \simeq 2 e \frac{m_{\mathrm{DM}}}{M_{1}^{2}} \sin \phi F\left(m_{i}, M_{1}, M_{2}\right),
$$

where $F$ is a smooth function of the internal charged lepton masses $m_{i}$ and Higgs masses $M_{1}$ and $M_{2}$, varying from 1 to 10 for reasonable values of the parameters. Within the experimental limits on neutrino mixing and Higgs boson masses that follow from negative neutrino oscillation [24] and Higgs boson searches [26] one can reach values up to $3 \times 10^{-14} \mu_{\mathrm{B}}$ for $m_{\mathrm{DM}}=30 \mathrm{eV}$.

In order to account for the photon flux ionizing interstellar hydrogen one should have $M \sim 30 \mathrm{eV}$ and $\mu \sim 10^{-14} \mu_{\mathrm{B}}$. It is easy to verify that both requirements can easily be met in our model. However, a $30 \mathrm{eV}$ hot dark matter neutrino made out of two active components, $\nu_{\mu}$ and $\nu_{\tau}$, may cause problems with several observations. For example, the ratio of the neutrino density to the critical density is determined from

$$
\Omega_{\nu}=\frac{\sum m_{\nu}}{h^{2} 91 \mathrm{eV}}
$$

where $h$ is the (dimensionless) Hubble constant. If we require that the density of the universe be the critical density, as suggested by inflation, the age of the universe is given by

$$
t=\frac{2}{3 H}
$$


Two $30 \mathrm{eV}$ neutrinos would then imply that the age of the universe to be $8 \mathrm{Ga}$, which contradicts the observational lower limit $10 \mathrm{Ga}$. In principle this age problem could be avoided by relaxing the requirement that $\Omega=1$. One could have acceptable ages of more than ten billion years with $\Omega \gtrsim 3$.

Models with only hot dark matter also have difficulties to explain the structures observed in the universe. These could be corrected by imposing strong bias on these schemes, as could arise from topological defects in the very early universe, e.g. cosmic strings. These could produce substantial seeds for galaxy formation while the galactic halos can be explained by baryonic matter. In fact there seems to be some evidence that HDM plus cosmic strings has roughly the properties that seem to be required for galaxy formation and is in agreement with CORE results [21].

\section{Rare decays}

Our choice of the symmetry group allows tau leptons to decay to final states involving electron and something else. With the couplings defined in the lagrangian, the fastest new decay mode would be the decay to two electrons and a positron. It occurs through the tree-level graph of fig. 2, and its branching ratio is given by

$$
\mathrm{BR}(\tau \rightarrow 3 \mathrm{e}) \simeq \frac{g_{\mathrm{e} \tau}^{2} g_{\mathrm{ee}}^{2}}{g_{2}^{4}} \frac{M_{\mathrm{W}}^{4}}{M_{1}^{4}} .
$$

The experimental constraints of our model allow this to be as large as the present experimental limit $\left(4 \times 10^{-5}\right)$ [24], which makes it reasonable to search for this process in future tau factories. The most obvious choice of the parameters providing the solutions to dark matter, solar neutrino and atmospheric neutrino problems yield branching ratios inaccessible to any feasible experiment, but there is a certain completely natural region of parameters giving observable rates while at the same time reconciling the above problems. For example, our previous sample choice gives $\operatorname{BR}(\tau \rightarrow 3 \mathrm{e}) \sim 3 \times 10^{-6}$.

At one-loop level one can have radiative tau decays. For a certain range of parameters this can also be observable, but it is likely to be smaller than the decay to electrons. The branching ratio is given by

$$
\mathrm{BR}(\tau \rightarrow \mathrm{e}+\gamma) \simeq 4 \times 10^{5} g_{\mathrm{e} \tau}^{2} g_{\tau \tau}^{2}\left(\frac{\mathrm{GeV}}{M}\right)^{4} .
$$

If we choose to break the symmetry spontaneously, the tau can decay into an electron and a majoron, with a branching ratio

$$
\mathrm{BR}(\tau \rightarrow e+J) \simeq 5 \times 10^{6} g_{\mathrm{e} \tau}^{2} g_{\tau \tau}^{2} \frac{M_{\kappa}^{4}\langle\sigma\rangle^{2}}{\bar{M}^{\circ}}
$$



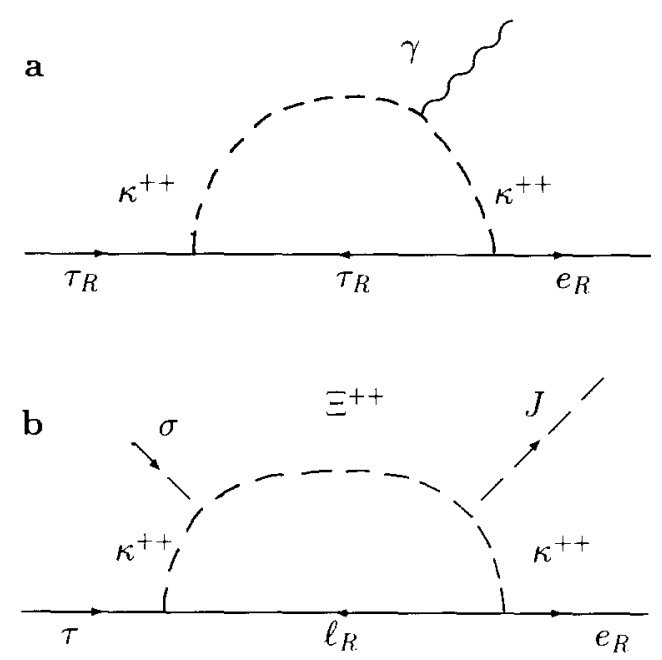

C

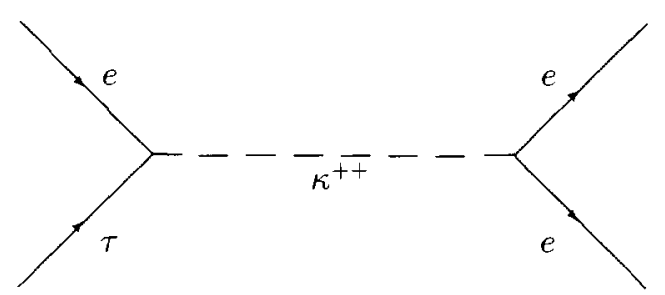

Fig. 2. Diagrams responsible for the decays $\tau \rightarrow \mathrm{e}+\mathrm{J}$ and $\tau \rightarrow \mathrm{e}+\gamma$ and $\tau \rightarrow 3 \mathrm{e}$.

where $M_{\kappa}$ is the cubic $\kappa \Xi \sigma$ coupling constant and all masses are expressed in $\mathrm{GeV}$. For the above parameter choice and similarly for any other natural choice that reconciles all the three problems this is expected to be below experimental detectability. However, relaxing the atmospheric neutrino condition the majoronic decay could be as large as the current search limit.

All other rare decays, especially those involving muons, are more suppressed, as they require the violation of our global symmetry.

\section{The $e-\tau+\mu$ model}

An entirely similar, physically inequivalent model can be obtained by replacing the underlying global symmetry $\mathrm{e}-\mu+\tau$ by the $\mathrm{e}-\tau+\mu$ symmetry. For this case 
we can apply the previous formulae, with $\mu$ and $\tau$ interchanged. A very important difference is that now the most relevant mixing angle is that between electrons and muons, which is given by

$$
\tan \theta \simeq \frac{m}{M} \simeq \frac{f_{\tau \mathrm{e}}}{f_{\mu \tau}}
$$

which controls the rates for $\nu_{\mu}$ to $\nu_{\mathrm{e}}$ oscillations at accelerators. Existing laboratory experiments restrict this mixing angle to $\sin ^{2} 2 \theta<0.004$ [24], which gives for the coupling constants a bound $f_{\mathrm{e} \tau}<0.03 f_{\mu \tau}$.

One can easily generate a mass matrix that satisfies our previous dark matter, solar and atmospheric neutrino conditions, for example with the choice $f_{\mathrm{e} \tau}=10^{-5}$, $f_{\mu \tau}=4 \times 10^{-4}, g_{\mathrm{ee}}=6 \times 10^{-3}, g_{\mu \mathrm{e}}=3 \times 10^{-4}, g_{\mu \mu}=0.2, \xi_{\mathrm{e}}=10^{-5}, \xi_{\mu}=2 \times 10^{-5}$, $M_{\mathrm{s} \phi \bar{\phi}}=5 \mathrm{GeV}, M_{\kappa} \equiv=100 \mathrm{GeV}, M_{\mathrm{s} \eta \kappa}=M_{\eta \eta \Xi}=170 \mathrm{GeV}, M_{0}=160 \mathrm{GeV}, v=80$ $\mathrm{GeV}, \tilde{v}=240 \mathrm{GeV}, \sin \beta=0.2$. On the other hand, it is more difficult to generate sizeable magnetic transition moments, because of the more stringent bound for the mixing of electron and muon neutrinos, and less favourable integral factors.

The estimated branching ratios of lepton flavour violating muon decays and their present experimental limits [24] are given by

$$
\begin{gathered}
\mathrm{BR}(\mu \rightarrow 3 \mathrm{e}) \simeq \frac{g_{\mathrm{e}, g_{\mathrm{ee}}^{2}}^{2}}{g_{2}^{4}} \frac{M_{\mathrm{w}}^{4}}{M_{1}^{4}} \leq 10^{-12}, \\
\mathrm{BR}(\mu \rightarrow \mathrm{e}+\gamma) \simeq 4 \times 10^{5} g_{\mathrm{e} \mu}^{2} g_{\mu \mu}^{2}\left(\frac{\mathrm{GeV}}{M}\right)^{4} \leq 5 \times 10^{-11}, \\
\operatorname{BR}(\mu \rightarrow \mathrm{e}+J) \simeq 10^{9} g_{\mathrm{e} \mu}^{2} g_{\mu \mu}^{2} \frac{M_{\kappa}^{4}\langle\sigma\rangle^{2} \mathrm{GeV}^{2}}{M^{8}} \leq 2.6 \times 10^{-6},
\end{gathered}
$$

where the last process exists only if we have the spontaneous violation of the underlying global symmetry. Similar values can been found in the literature for the first two modes [25]. One can easily verify that all these rates can reach values within the sensitivities of present and future experiments. Our sample set of values gives $\sim 4 \times 10^{-13}$ for the case of $\mu \rightarrow 3 \mathrm{e}, \sim 2 \times 10^{-12}$ for $\mu \rightarrow \mathrm{e} \gamma$ decays and $\sim 2 \times 10^{-6}$ for the majoron emitting decay mode (choosing $\langle\sigma\rangle=170 \mathrm{GeV}$ ). It is quite possible to obtain value of these branching ratios in excess of present limits [24]!

\section{Discussion}

We have analyzed interesting symmetries of the leptonic electroweak interaction which are suggested in order to reconcile the solar and atmospheric neutrino 
data with the hot dark matter component in the universe. This requires the presence of a fourth light neutrino species $\nu_{\mathrm{s}}$, singlet under the $\mathrm{SU}(2) \otimes \mathrm{U}(1)$ symmetry. We have also provided concrete models that embody this symmetry and in which the HDM is a quasi-Dirac neutrino formed by $\nu_{\mu}$ and $\nu_{\tau}$ whose mass $m_{\mathrm{DM}}$ is induced at the one-loop level. Unlike a previous suggestion, in the present model the sterile neutrino is ultralight, and the solar neutrino deficit is explained via nonadiabatic conversions of $\nu_{\mathrm{e}}$ to $\nu_{\mathrm{s}}$, while the atmospheric neutrinos are explained via maximal $\nu_{\mu}$ to $\nu_{\tau}$ oscillations. Both oscillations are generated as breaking effects and arise as higher order loop diagrams which can naturally provide the required small mass splittings.

For $m_{\mathrm{DM}} \sim 30 \mathrm{eV}$ the radiative HDM decay leads to photons that can ionize interstellar hydrogen. The price is that, for $\Omega=1$, we have a relatively young universe with $8 \mathrm{Ga}$. The complete inner consistency of this possibility from the particle physics point of view and its ability to explain other astrophysical and cosmological puzzles in an attractive way highlight, in our opinion, the interest in further pushing on the determination of the age parameter.

The $\nu_{\mathrm{e}}$ to $\nu_{\tau}$ oscillations can have experimentally observable rates, with no appreciable $\nu_{\mu}$ oscillations expected at accelerator experiments. In addition, this model can lead to tau number violating processes such as $\tau \rightarrow \mathrm{e}+\gamma, \tau \rightarrow \mathrm{e}+\mathrm{J}$ and $\tau \rightarrow 3 \mathrm{e}$ with rates than can lie within the sensitivities of future tau factories.

In the variant model described in sec. 6 the oscillations that are phenomenologically relevant for accelerator experiments are $\nu_{\mathrm{e}}$ to $\nu_{\mu}$, and they can have experimentally observable rates. In this case the lepton flavour violating processes involve muons, e.g. $\mu \rightarrow 3 \mathrm{e}, \mu \rightarrow \mathrm{e}+\gamma$ and $\mu \rightarrow \mathrm{e}+\mathrm{J}$. Their rates can lie within the sensitivities of present experiments.

We stress that these models are compatible with laboratory, astrophysical and cosmological observations, including primordial nucleosynthesis limits. The new Higgs bosons present in these models also modify the baryogenesis conditions [22]. Conventional models for the generation of the baryon asymmetry within the standard model are likely to contradict [23] the laboratory limits for the Higgs boson masses [26]. These constrains may be avoided in multi-Higgs models, like ours. We can also have large $C P$ violation either on the Yukawa couplings, or in the Higgs sector. These effects would not be restricted by the physics of the quark sector, as our new Higgses do not couple to quarks.

This work was supported by the Spanish Ministry for Education and Science. We thank David Caldwell, Vadim Kuzmin, Anjan Joshipura and Daniele Tommasini for discussions, and Adrian Mellott and Dennis Sciama for bringing to our attention the literature on unstable dark matter and the ionization of interstellar hydrogen. 


\section{References}

[1] G.F. Smoot et al., Ap. J. 396 (1992) L1;

E.L. Wright et al., Ap. J. 396 (1992) L13; Berkeley preprint (1992);

R. Schaefer and Q. Shafi, Phys. Rev. D47 (1993) 1333;

G. Estathiou et al., Oxford OXAST/92/11 (1992);

M. Davis et al., Berkeley CfPA-TH-92-016 (1992)

[2] J.R. Davis et al., in Proc. 21th Int. Cosmic Ray Conf. Vol. 12, ed. R.J. Protheroe (University of Adelaide Press, 1990) p. 143

[3] P. Anselmann et al., Phys. Lett. B285 (1992) 376, 390

[4] A. Abazov et al., Phys. Rev. Lett. 67 (1991) 3332

[5] K. Hirata et al., Phys. Rev. Lett. 65 (1990) 1297; 66 (1991) 9; Y. Suzuki, Int. Sym, on Neutrino astrophysics, Takayama (Oct. 1992)

[6] K. Hirata et al., Phys. Lett. B205 (1988) 416; B280 (1992) 146;

R. Becker-Szendy et al., Phys. Rev. D46 (1992) 3720; Phys. Rev. Lett. 69 (1992) 1010

[7] J.N. Bahcall and M.H. Pinsonneault, Rev. Mod. Phys. 64 (1992) 885; J.N. Bahcall and R.N. Ulrich, Rev. Mod. Phys. 60 (1988) 297;

S. Turck-Chièze, S. Cahen, M. Cassé, and C. Doom, Astrophys. J. 335 (1988) 415;

S. Turck-Chièze, Saclay preprint $1992 / 32$ (1992)

[8] See e.g. W. Frati, T.K. Gaisser, A.K. Mann and T. Stanev, Pennsylvania UPR-0218E (1992); G. Barr, T.K. Gaisser and T. Stanev, Phys. Rev. D39 (1989) 3532

[9] D.K.S. Bludman and P. Langacker, Nucl. Phys. B374 (1992) 373; S.A. Bludman, D.C. Kennedy and P.G. Langacker, Phys. Rev. D45 (1992) 1810; Nucl. Phys. B373 (1992) 498

[10] E.L. Wright et al., Ap. J. 396 (1992) L13; M. Davis, E.J. Summers and D. Schlegel, Nature 359 (1992) 393; A.N. Taylor and M. Rowan-Robinson, Nature 359 (1992) 396; J. Holtzmann, Ap. J. Suppl. 71 (1989) 1

[11] A. de Rújula and S.L. Glashow, Phys. Rev. Lett. 45 (1980) 942; A.L. Melott and D.W. Sciama, Phys. Rev. Lett. 46 (1981) 1369; P.B. Pal and L. Wolfenstein, Phys. Rev. D25 (1982) 766; A.L. Melott, D.W. McKay and J.P. Ralston, Ap. J. 324 (1988) L43; D.W. Sciama, Nucl. Phys. B (Proc. Suppl.) 19 (1991) 138; G.J. Hogan, Nature 359 (1992) 40

[12] N. Armenise et al., CERN preprint CERN-SPSC/90-42 (1992); P. Astier et al., CERN preprint CERN-SPSC/91-21 (1992);

K. Kodama et al, FNAL preprint proposal P803 (1991)

[13] J.T. Peltoniemi, D. Tommasini and J.W.F. Valle, Phys. Lett. B298 (1993) 383; L. Bento and J.W.F. Valle, Phys. Lett. B264 (1991) 373

[14] A. Smirnov and J.W.F. Valle, Nucl. Phys. B375 (1992) 649; J.T. Peltoniemi, A. Smirnov and J.W.F. Valle, Phys. Lett. B286 (1992) 321

[15] K. Babu, R.N. Mohapatra and I. Rothstein preprint Phys. Rev. D45 (1992) R5; C.P. Burgess, J.M. Cline and M.A. Luty, Phys. Rev. D46 (1992) 364

[16] R. Barbieri and A. Dolgov, Nucl. Phys. B349 (1991) 742; K. Enqvist, K. Kainulainen and M. Thomson, Nucl. Phys, B373 (1992) 498; Phys. Lett. B280 (1992) 245

[17] A. Zee, Phys. Lett. B93 (1980) 389;

S. Petcov, Phys. Lett. B110 (1982) 245;

S. Bertolini and A. Santamaria, Nucl. Phys. B310 (1988) 714

[18] K.S. Babu and V.S. Mathur, Phys. Lett. B196 (1987) 218

[19] J.E. Kim, Phys. Rev. 150 (1987) 1 and references therein

[20] J. Schechter and J.W.F. Valle, Phys. Rev. D24 (1981) 1883; Phys. Rev. D25 (1982) 283

[21] A. Albrecht and A. Stebbins, Phys. Lett. 69 (1992) 2615

[22] G.W. Anderson and L.J. Hall, Phys. Rev. D45 (1992) 2685; 
J.T. Peltoniemi and J.W.F. Valle, Phys. Lett. B304 (1993) 147;

A.I. Bocharev, S.V. Kuzmin and M.E. Shaposnikov, Phys. Lett. B244 (1990) 275; Phys. Rev. D43 (1991) 369;

N. Turok and J. Zadrozny, Nucl. Phys. B358 (1991) 471;

B. Kastening, R.D. Peccei and X. Zhang, Phys. Lett B266 (1991) 413;

L. McLerran et al., Phys. Lett. B256 (1991) 451;

A.G. Cohen, D.B. Kaplan and A.E. Nelson, Phys. Lett. B245 (1990) 561; Nucl. Phys. B349 (1991) 727 ;

Y. Kondo et al. Phys. Lett. B263 (1991) 93; N. Sei et al., Phys. Lett. B299 (1993) 286

[23] V.A. Kuzmin, V.A. Rubakov and M.E. Shaposhnikov, Phys. Lett. 36 B155 (1985);

M. Dine, R.L. Leigh, P. Huet, A. Linde and D. Linde, Phys. Lett. B283 (1992) 319

[24] Particle Data Group, Phys. Rev. D45 (1992) S1, and references therein

[25] G. Leontaris et al., Phys. Lett. B162 (1985) 153

[26] D. Decamp et al. (ALEPH Collaboration), Phys. Lett. B236 (1990) 233; B237 (1990) 291; B241 (1990) 141; B245 (1990) 289; B246 (1990) 306; preprints CERN-PPE/91-149; P. Abreu et al. (DELPHI Collaboration), Nucl. Phys. B342 (1990) 1; Z. Phys, C51 (1991) 25; preprint CERNPPE/91-132;

B. Adeva et al. (L3 Collaboration), preprints CERN-PPE/92-40 and references therein;

M.Z. Akrawy et al. (OPAL Collaboration), Phys. Lett. B236 (1990) 224; B251 (1990) 211; B253 (1991) 511;

P.D. Acton et al. Phys. Lett. B268 (1991) 122 